\title{
Impact of rain weather over free space optic communication transmission
}

\author{
A.K Rahman ${ }^{1}$, N Julai ${ }^{2}$, C.B.M.Rashidi ${ }^{3}$, Nurdiani Zamhari ${ }^{4}$, S.K Sahari ${ }^{5}$, N.A.A Mohtadzar ${ }^{6}$, \\ M.R.M Sharip ${ }^{7}$ \\ ${ }^{1,2,4,5,6,7}$ Faculty of Engineering, Department of Electrical Electronic, University Malaysia Sarawak, Malaysia \\ ${ }^{3}$ Advance Communication Engineering, Centre of Excellence, SCCE, University Malaysia Perlis, Malaysia
}

\begin{tabular}{l}
\hline Article Info \\
\hline Article history: \\
Received Sep 27, 2018 \\
Revised Nov 26, 2018 \\
Accepted Nov 5, 2018 \\
\hline
\end{tabular}

\section{Keywords:}

Attenuation

Free space optic

Rain

Scattering effect

\begin{abstract}
This paper focuses the rain effect over FSO link medium in Malaysia environment. In this work, a rain data samples that collected from Malaysian Meteorological Department (MMD) to determine the scattering coefficient, atmospheric attenuation and total attenuation. From the analysis, the precipitation rate give different impact over FSO link which can cause the attenuation and bit error rate increase. The results also show the comparison parameter for optimal geometrical loss such as beam divergence, aperture size and receiver sensitivity.
\end{abstract}

Copyright (C) 2019 Institute of Advanced Engineering and Science. All rights reserved.

\section{Corresponding Author:}

Abdul Rahman Bin Kram,

Department of Electrical and Electronic Engineering,

University Malaysia Sarawak,

Fakulti Kejuruteraan, Jalan Dato Mohd. Musa, 94300 Kota Samarahan, Sarawak, Malaysia.

Email: karahman@unimas.my

\section{INTRODUCTION}

In telecommunications, Free Space Optics (FSO) which utilizes light propagation in free space to transmit data between two points has become an attractive alternative to optical fiber communication or radio frequency system [1]. Various applications can be applied in FSO systems, such as the last mile high bandwidth internet connectivity, the temporary high bandwidth data links, the mobile telephony backhaul $(3 \mathrm{G})$, satellite links as well as the various applications where the optical fibers cannot be used. However FSO communication is vulnerable to atmospheric weather.

There are several factors which contribute to the degradation of FSO performance, which includes scintillation, absorption and scattering [2], [3]. In propagation of optical signals, the performance changes even more easily, especially in varying weather effects. Fog, rain, dust, snow, smoke, and other aerosol particulate matter primarily attenuate the signal-carrying laser beam [4].

This work focuses on attenuation due to rainfall, which in turn generates the scattering effect. Scattering is caused by particles such as fog, haze and rain. The type of scattering is determined by the size of the source particles. Scattering caused by particles which are equal or larger compared to the transmitted light wavelength is referred to as Mie scattering. To analyze the scattering rate in these cases, it is more appropriate to use geometric optics. In scattering, energy is taken from the beam when the beam encounters particles or molecules of different sizes and shapes. Thus, the main atmospheric attenuation component was assumed to be only of scattering losses. Other factors such as dust or liquid particles in air could also produce scattering, given that there are irregularities on the signal-entry surface. 
Previous researchers have been investigating to determine the level of attenuation due rainfall, and relating it to the rain rate [5], [6], [7], [8]. However, most of the researches were focused on European weather, and minimal effort has been channeled to investigate this effect in the tropical region, which experiences a significant amount of rainfall. An almost-daily rainfall which totals up to between 1500 and $2000 \mathrm{~mm}$ annually, was certain to influence the quality transmission of FSO link deployed. This effort will address this vacancy to a significant extent, with precise mathematical relations, derived and improved over the years by various researchers [9] coupled by simulations using OptiSystem software.

\section{RESEARCH METHOD}

A. Theoretical

The attenuation of laser power by rain can be calculated and is best described by Beer's law [10], [11]:

$$
\tau(R)=\frac{P(R)}{P(0)}=e^{-\beta R}
$$

where $\mathrm{R}$ is the link range in meters, $\tau(\mathrm{R})$ is the transmittance at range $\mathrm{R}(\mathrm{km}), \mathrm{P}(\mathrm{R})$ is the laser power at range R. $\mathrm{P}(0)$ is the laser power at the source (Watt) and $\beta$ is the scattering coefficient $\left(\frac{1}{\mathrm{~km}}\right)$.

The scattering coefficient can be calculated by using Stroke Law [12]. The scattering coefficient due to rain with drops of radius $a$, is defines as:

$$
\beta_{\text {rainscat }}=n(\sigma)
$$

Where $n$ is the number of raindrops per unit volume for drops with radius $a$, and $\sigma$, the scattering cross section,

$$
(\sigma)=\pi a^{2}(Q)_{\text {scatt }}
$$

$(\mathrm{Q})_{\text {scat }}$ is the wavelength-dependent scattering efficiency from the Mie theory [13][14]. Now, for $\mathrm{x}=$ $2 \pi a / \lambda \gg 1,(Q)_{\text {scat }}$ approaches 2 as a limit [13], [14]. The average raindrops size is about $0.05 \mathrm{~cm}$. For infrared wavelengths of interest, e.g., $3-5 \mu$ or $8-15 \mu$, then $\mathrm{x} \approx 300-1000$; thus $(\mathrm{Q})_{\text {scat }}=2$. The scattering coefficient becomes

$$
\beta_{\text {rainscatt }} \cong n 2 \pi a^{2}
$$

It was found experimentally that the rain particle size distribution is a function of the total precipitation rate $\mathrm{Z}[15]$. $\mathrm{Z}_{\mathrm{a}}$ is related to $\mathrm{Z}$ in the following manner:

$$
z_{a}=\alpha z
$$

Where $\mathrm{Z}_{\mathrm{a}}$ is the rate of rainfall in cm (depth of water) per second for drops with radius $a$, and $\alpha$ is the fraction of total volume reaching ground contributed by drops of radius $a$. Since the measured values of the extinction coefficient due to rain are usually presented as a function of the total precipitation rate [16], it is useful to express the calculated coefficient in term of the same parameter. $\beta_{\text {rainscat }}$ can be related to $\mathrm{Z}_{\mathrm{a}}$ by first noting that.

$$
n=\frac{\rho z_{a} / v_{a}}{\rho V_{a}}
$$

Where $\rho=$ density of water, $2 \mathrm{gm} / \mathrm{cc}, \mathrm{V}_{\mathrm{a}}=$ terminal velocity of raindrops size $a, \mathrm{~cm} / \mathrm{sec}, \mathrm{V}_{\mathrm{a}}=$ volume of each raindrops of radius $a, 4 \pi \mathrm{a}^{3} / 3$

Substituting (6) into (4) yield

Indonesian J Elec Eng \& Comp Sci, Vol. 14, No. 1, April 2019 : 303 - 310 


$$
\beta_{\text {rainscatt }}=\frac{3}{2} \frac{Z_{a}}{v_{a}} \frac{1}{a} \mathrm{~cm}^{-1}
$$

Terminal velocity of a raindrop through air can be obtained by a Stroke Law:

$$
v=\frac{2 a^{2} \rho g}{9 \eta}
$$

Where, as before, $\rho=$ density of the droplet, $1 \mathrm{gm} / \mathrm{cc}, \mathrm{a}=$ radius of the raindrop, $\mathrm{cm}, \mathrm{g}=$ acceleration due to gravity, $980 \mathrm{~cm} / \mathrm{sec}^{2,} \eta=$ viscosity of the air, $180 \times 10^{-6}$ Substituting (8) into (7) will simply the expression for $\beta_{\text {rainscatt }}$ :

$$
\beta_{\text {rainscatt }}=1.25 \times 10^{-6}\left(\frac{Z_{a}}{a^{3}}\right) \mathrm{cm}^{-1}
$$

Another of significant loss due to atmospheric that must be taken into account is the geometrical spreading attenuation. Geometrical attenuation occurs when the transmitted beam spreads with increasing range. Even in clear conditions, the beam diverges and as result, the detector collects less signal power [17]. The geometric losses are simply the ratio of the surface area of the receive aperture to the surface area of the transmit beam at the receiver.

$$
\text { Geometrical Loss }(\mathrm{GL})=\frac{\pi r_{2}{ }^{2}}{\pi r_{1}{ }^{2}+\pi r_{3}{ }^{2}}
$$

where $r_{1}=$ radius of transmitter aperture, $r_{2}=$ radius of receiver aperture and $r_{3}=$ radius of beam area The geometric losses depend primarily on the divergence and the range [18].

$$
\mathrm{GL}=\frac{\pi\left(\frac{d_{2}}{2}\right)^{2}}{\pi\left(\frac{d_{1}}{2}\right)^{2}+\pi\left(\frac{d_{3}}{2}\right)^{2}}
$$

where $\mathrm{d}_{1}, \mathrm{~d}_{2}$ and $\mathrm{d}_{3}$ are diameters. Replace $\mathrm{d}_{3}$ with $\theta R$ will yield a final geometric loss expression.

$$
\text { Geometric losses }(\mathrm{GL})=\frac{d_{2}{ }^{2}}{\left[\left(d_{1}+(\theta R)\right]^{2}\right.}
$$

Therefore, the total attenuation expression can be derived. The total attenuation is a combination of atmospheric attenuation and geometric loss. The total attenuation for FSO system is actually very simple at a high level (leaving out optical efficiency, detector noises, etc). It can be described as a ratio of output power receive over power transmit which is equal to geometrical loss multiplied by transmittance at range R. The equation can be written as [19]:

$$
\frac{P_{\text {transmit }}}{\mathrm{P}_{\text {receive }}}=10 \log \frac{d_{2}^{2}}{\left(d_{1}+(\theta R)\right)^{2}} *\left(e^{\beta R}\right)
$$

where $d_{2}=$ diameter receiver aperture $(m), d_{1}=$ diameter transmitter aperture $(m), \quad=$ beam divergence $(\mathrm{mrad}), \mathrm{R}=$ link range $(\mathrm{km}), \quad=$ scattering coefficient $(1 / \mathrm{km})$.

B. Simulation

The simulation setup for an FSO communication system in order to implement atmospheric attenuation is shown in Figure 1. The continuous wave lasers were used to create a carrier signal. In this simulation, two types of lasers were used, with wavelengths of $785 \mathrm{~nm}$ and $1550 \mathrm{~nm}$. The carrier signal was then encoded through a multiplexer before modulated in an external Mach-Zehnder modulator. The pseudorandom bit sequence generator was used to generate the random input data bit sequence at the rate of $155 \mathrm{Mbps}$. 


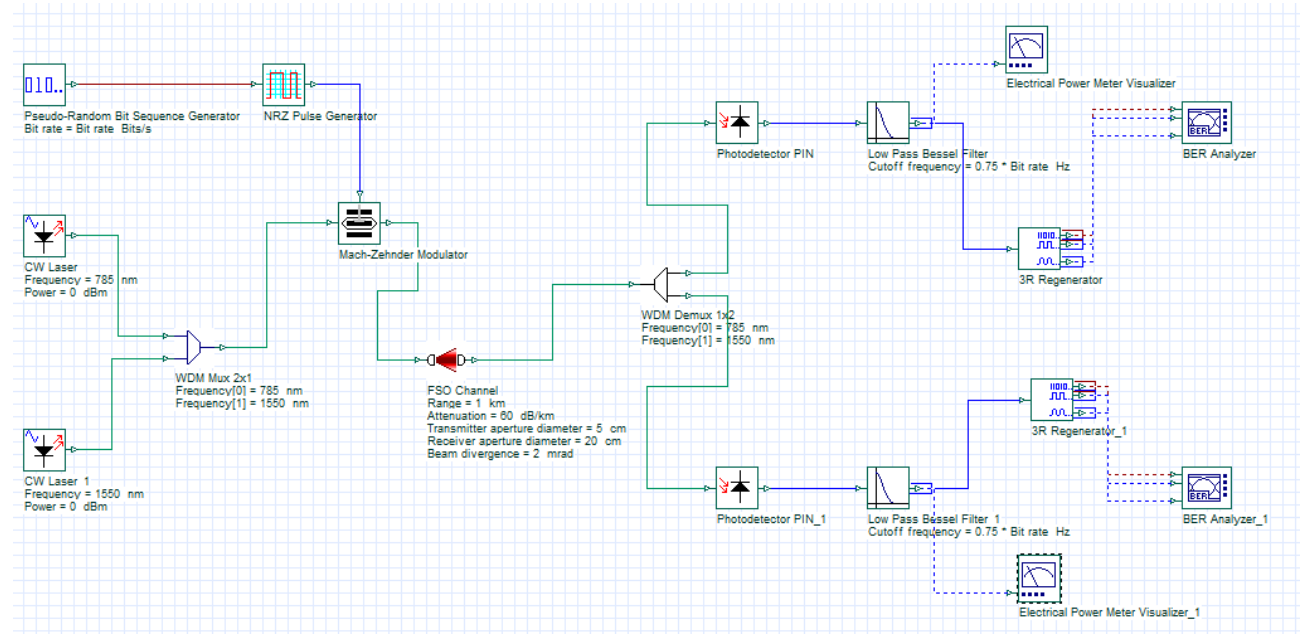

Figure 1. Free space optic simulation setup

After that, a non-return-to-zero (NRZ) pulse generator was used to act as the input for modulation in an external modulator. The modulated data was then transmitted via the free space optic (FSO) channel. In the FSO channel, the range was activated and was set to $1 \mathrm{~km}$. The attenuation considered was losses that were caused by atmospheric effect (rain and haze) from low to high attenuation $(10 \mathrm{~dB} / \mathrm{km}-60 \mathrm{~dB} / \mathrm{km})$.

Meanwhile, a geometrical loss was defined with a transmitter aperture size of $0.05 \mathrm{~m}$ and a receiver aperture size of. $0.25 \mathrm{~m}$. The beam divergence was set to $1 \mathrm{mrad}$. All attenuations, geometrical losses and beam divergence were specified according to typical industrial values in order to simulate as close as possible to the real environment.

The receiver side consists of a splitter, photodiode and filter. The splitter splits an optical input into two output signal back to channel wavelengths of $785 \mathrm{~nm}$ and $1550 \mathrm{~nm}$, respectively. The photodiode was used for optical to electrical conversion before signal flow to a low pass filter. The decoded signal will then arrive at signal inspection equipment such as the BER analyzer and Eye-Diagram analyzer. The received power was measured using Optical Power Meter.

\section{RESULTS AND ANALYSIS}

Figure 2 shows the total attenuation corresponding to the rainfall rate. Two designs with different transmitter and receiver aperture sizes were compared - Design 1 and Design 2. Both aperture design dimensions were defined based on commercial FSO system that is widely available in market today. It can be seen from the graph that Design 1 was performing better than Design 2, producing a lower attenuation level.

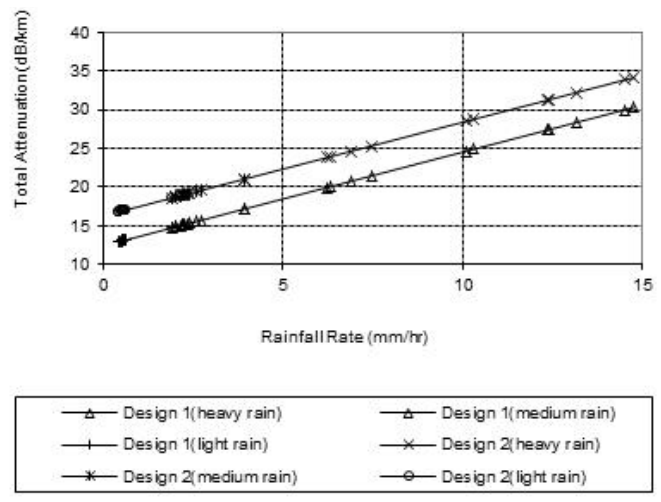

Figure 2. Total attenuation versus rainfall rate 
As the receiver diameter increases, the geometric loss was reduced, comparing to small receiver. This is because the larger collecting area aperture significantly reduces signal fluctuation by averaging the received waveform over the aperture area. Therefore, in heavy rain conditions which produce higher total attenuation, the deployment of a small transmitter and larger receiver aperture will help in improving the link.

Table 1 shows the prediction of the maximum allowable rainfall rate which could impair the FSO link, against different link distances. The receiver sensitivity was assumed to have a BER of $10^{-9}$ and Design 1 was analyzed. It can be seen that with fixed receiver sensitivity and at short distance, a higher rainfall rate was able to be tolerated by the system. As the link distance increases, the tolerable level of rainfall rate will drop, due the receiver's inability to receive sufficient signal power. This prediction is significant in order to determine the maximum laser traveling distance and its corresponding rainfall rate limit.

Figure 3 shows the received power due to the total atmospheric attenuation over system's link. It can be seen that for both wavelengths, the received power uniformly decreased with the increase in total atmospheric attenuation. The wavelength $1550 \mathrm{~nm}$ was found to receive a higher power level, as the longer wavelength was able to penetrate attenuating particles, therefore, experiencing lower power loss.

One of the factors discussed previously, in reducing atmospheric attenuation effect was the application of a narrow optical beam. Figure 4 shows the consequence of the beam divergence, by investigating the received power against the total atmospheric effect, for both wavelengths of $785 \mathrm{~nm}$ and $1550 \mathrm{~nm}$. It can be seen that all received power decreased constantly with an increased attenuation, with different beam divergence. The beam divergence of $1 \mathrm{mrad}$ was found to be receiving the maximum power when transmitted at $1550 \mathrm{~nm}$. A wide beam divergence will cause a high power loss into the atmosphere during transmission. On the other hand, a narrow beam tends to focus transmission signal and minimize power loss, thus, higher received power.

Table 1. The Prediction of Maximum Rainfall Rate at Different Link Range

\begin{tabular}{llllll}
\hline Range $(\mathrm{Km})$ & 0.5 & 1 & 1.5 & 2 & 2.5 \\
\hline $\begin{array}{l}\text { Maximum } \\
\text { Rainfall } \\
(\mathrm{mm} / \mathrm{hr})\end{array}$ & 155 & 70 & 40 & 30 & 20 \\
$\begin{array}{l}\text { Receiver } \\
\text { Sensitivity }\end{array}$ & $-42 \mathrm{dBm}(\mathrm{APD}$ commercially type) & \\
$\begin{array}{l}\text { BER }=10^{-9} \\
\text { Aperture }\end{array}$ & Transmitter $=0.05 \mathrm{~m}$ & $\begin{array}{l}\text { Receiver }= \\
0.25 \mathrm{~m}\end{array}$ \\
\hline
\end{tabular}

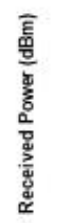

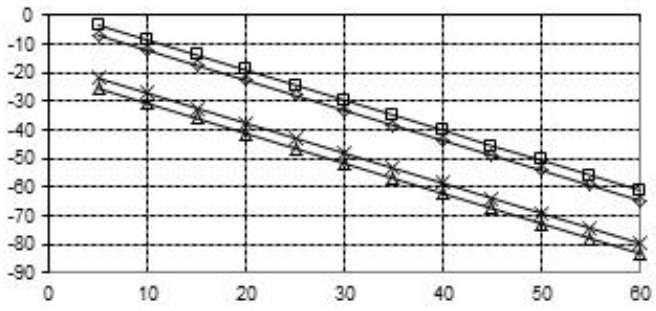

Attenuation $(\mathrm{dB} / \mathrm{km})$

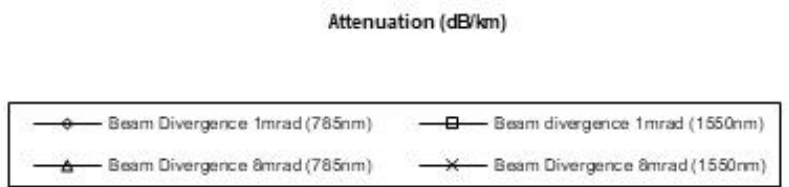

Figure 3. Received power versus total atmospheric attenuation 


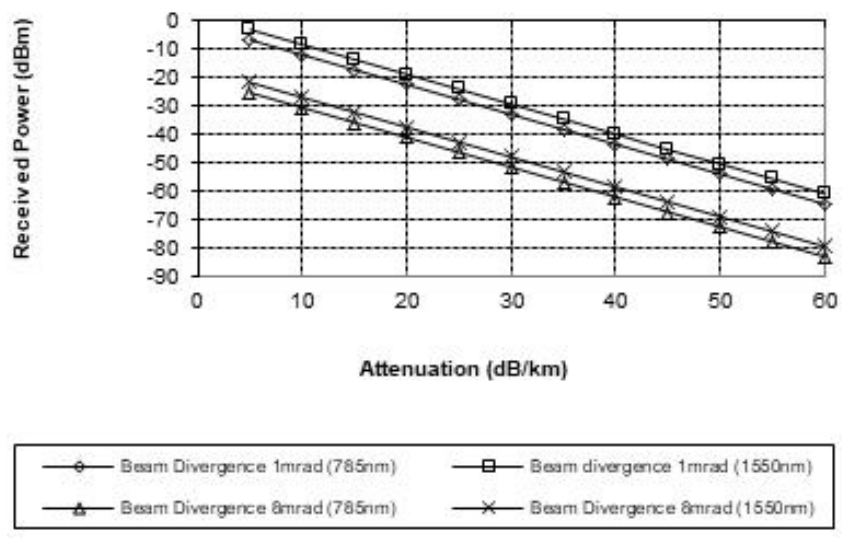

Figure 4. Received power versus attenuation for different beam divergence

Figure 5 shows received power versus the total atmospheric attenuation for different aperture sizes. The transmitter/receiver aperture sizes for both Design 1 and Design 2 were as discussed previously. All received power uniformly decreased with attenuation increase, and the best performing setup was Design 1 transmitting on a 1550nm wavelength. The clear advantage of a larger receiver area was its ability to reduce errors due to scintillation, which is an atmospheric turbulence due to solar loading and natural convection. It causes temporally and spatially varying refractive index changes in the air, time-varying a laser beam's propagation through the atmosphere. This phenomenon is also quite similar to the apparent twinkling of the stars or distant city lights. The result is that an FSO communications receiver can experience error bursts due to surges and fades in the receive signal strength. Utilizing a receiver aperture which is relatively much larger than the spatial scale of the scintillation provides an averaging effect of the localized surges and fades, thus improving the error rate. With the reduction of this effect the total attenuation also can be decreased.

Figure 6 shows the BER versus attenuation for different photo detector at the receiver. The graph is represented by two detector APD and $\mathrm{p}-\mathrm{i}-\mathrm{n}$ detector, for a single operating wavelength (1550nm). According from the graph, the attenuation at $-35 \mathrm{~dB} / \mathrm{km}$ the performance of APD photodiode detector was found to be better than a p-i-n detector. The BER value for APD was of the order $10^{-32}$ whereas a p-i-n detector, on the other hand, produced BER in the order of $10^{-05}$, which made the APD a more popular choice for FSO equipment vendors in their designs. This is due to the high level of attenuation which exists in FSO communication systems. Using detectors with higher sensitivity increases the ability of a receiver to detect lower power levels and maintain transmission quality within high atmospheric attenuation conditions. This condition can be observed deeply in eye pattern in figure below between 2 different type detectors. A large eye opening and greater height level represents the link's good condition, whereas a smaller eye opening indicates the links proneness to error.
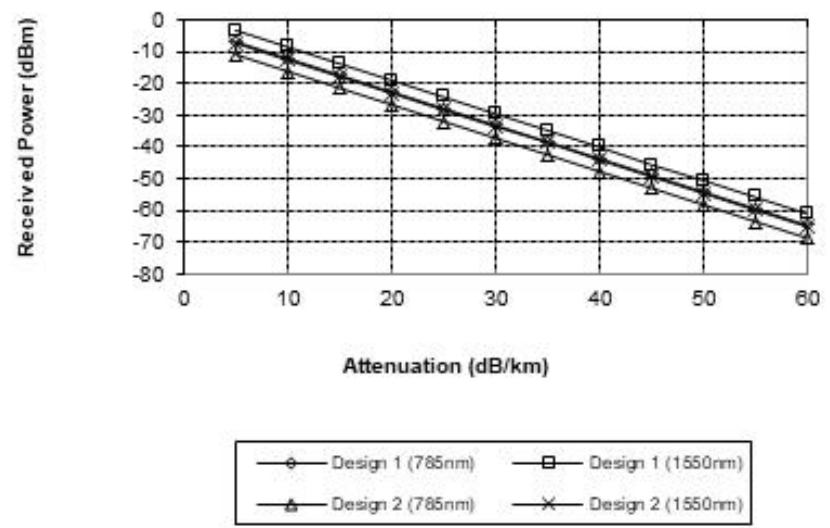

Figure 5. Received power versus attenuation for different aperture size 


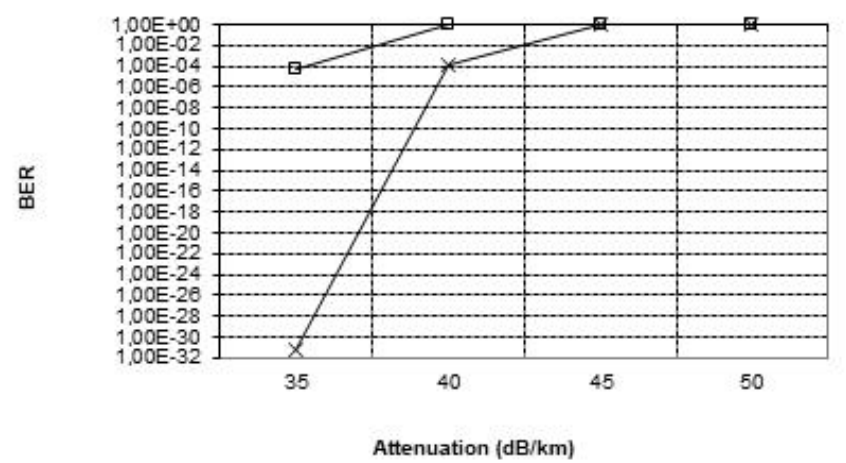

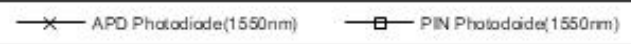

Figure 6. BER versus attenuation for different photo detector at receiver

Figure 7 and 8 shows the eye diagram for both APD and p-i-n for a constant attenuation of $35 \mathrm{~dB} / \mathrm{km}$. It can be seen that the opening eye pattern for $\mathrm{p}-\mathrm{i}-\mathrm{n}$ was small, with an eye height of $2.43 \times 10^{-7}$, whereas the opening eye pattern for APD was slightly better, with a height at $2.49 \times 10^{-6}$. The BER values for APD and p-i-n photo detector were found to be $5.26 \times 10^{-5}$ and $6.17 \times 10^{-32}$. It showed that at $-35 \mathrm{~dB} / \mathrm{km}$ the link with the p-i-n type detector was performing with high errors, as the minimum BER limit should be in the order of $10^{-9}$. On the contrary, the link with an APD detector produced a much lower BER and operating under good conditions.

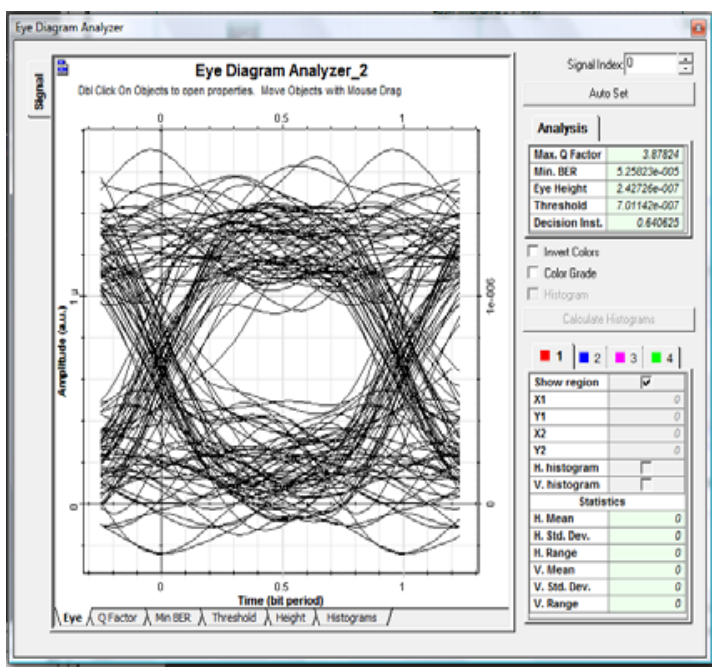

Figure 7. Eye diagram pattern at attenuation $35 \mathrm{~dB} / \mathrm{km}$ for $\mathrm{p}-\mathrm{i}-\mathrm{n}$ photodiode

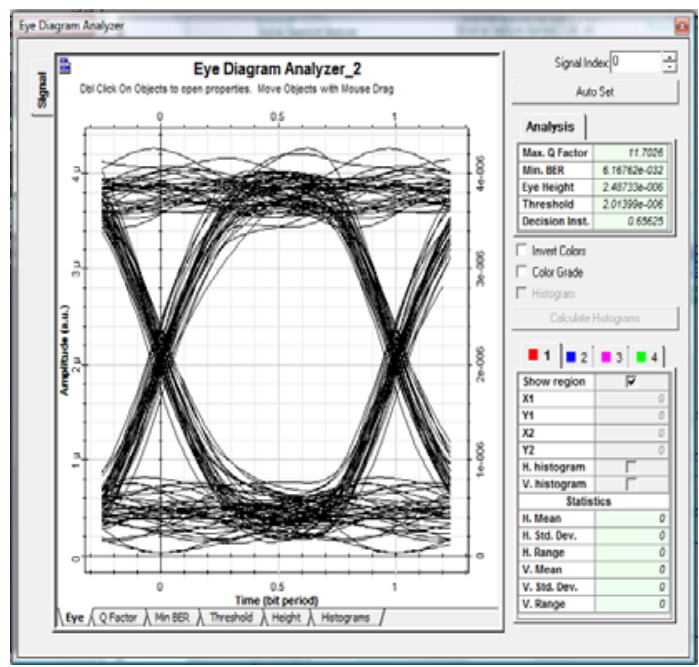

Figure 8. Eye diagram pattern at attenuation $35 \mathrm{~dB} / \mathrm{km}$ for APD photodiode

\section{CONCLUSION}

In this work, the study of rain effect upon an FSO communication system in the tropical region was presented. The result shows that the rain in Malaysia environment can effect the transmission FSO especially for heavy rain condition. The attenuation can deteriorate link approximately $60 \mathrm{~dB} / \mathrm{km}$. Based on simulation for optimal performance, the parameter can be used apply by using narrow beam which refer to small transmitter. For receiver is better larger size to collect more light. In term of operating wavelength, the longer wavelength $1550 \mathrm{~nm}$ window is much better due to penetration ability. Meanwhile at receiver part APD photodetector is highly sensitive compare to PIN detector. As result receiver can detect a weak signal to increase power received. 


\section{ACKNOWLEDGEMENTS}

This work has been supported by MyRA grant Universiti Malaysia Sarawak.

\section{REFERENCES}

[1] A. Akbulut, H. G. Ilk and F. Ari, Design, Availability and Reliability Analysis on an Experimental Outdoor FSO/RF Communication System, IEEE, p.(2005).

[2] Robert M.agliardi Sherman karp, Optical Communications, John Wiley \& Sons, 1995.

[3] Karp Gagliardi Moran Stotts, Optical Channels Fibers, Clouds, water, and the Atmosphere, Plenum Press, 1988

[4] M.Gebhart, E.Leitgeb, J.Bregenzer, Atmospheric effects on Optical Wireless links, ConTEL2003, Vol.2, pp.395 401, April 2004.

[5] M. Achour, Simulating atmospheric free-space optical propagation: rainfall attenuation, Free-Space Laser Communication Technologies XIV, Proc. SPIE, vol. 4635, pp.192-201, April 2002.

[6] J.T.Ong, K.I. Timothy, Chong \& S.V.B.Rao, Heavy rain effects on the propagation of free space optical links in Singapore, ICAP 2003, (Conf. Publ. No. 491),Vol.1, pp. 365 - 368, April 2003.

[7] Vikas Kukshya, T.S.Rappaport, H. Izadpanah, G. Tangonan, R.A. Guerrero, J.K. Mendoza and Brey Lee, FreeSpace Optics \& highspeed RF for Next Generation Networks-Propagation Measurements, VTC2002 IEEE 56th Vol.1, pp.616 - 620, 2002.

[8] [8]Haiping Wu, Hamzeh.B, Kavehrad, M, Achieving carrier class availability of FSO link via a complementary RF link, Systems and Computers, Conference Record of the Thirty-Eighth Asilomar Conference Vol.2, pp.1483 - 1487, 2004.

[9] Haiping Wu Achieving Carrier Class Availability of FSO Link via a Complementary RF Link Signals, Systems and Computers. Conference Record of the Thirty-Eighth Asilomar Conference on, 2004.

[10] I. I. Kim, J. Koontz, H. Hakakha, P. Adhikari, R. Stieger, C. Moursund, M. Barclay, A. Stanford, R. Ruigrok, J. Schuster, and E. Korevaar, Measurement of scintillation and link margin for the TerraLink laser communication system, Wireless Technologies and Systems: Millimeter Wave and Optical, Proc. SPIE, Vol. 3232, pp. 100-118, 1997.

[11] D. Johnson: Optical Through the Air Communications Handbook, http://www.imagineeringezine.com, 2006.

[12] Achour,M. Simulating Atmospheric Free Space Optical Propagation Part 1, Rainfall Attenuation Proceeding of SPIE Vol.4635, 2002.

[13] Van de Hulst, H. G., Light Scattering by Small Particles, John Wiley and Sons, Inc., New York, 1957.

[14] Diermendjian, D., Electromagnetic Scattering on Spherical Poly-dispersions, The Rand Corporation, R-456-(April); also American Elsevier Publishing Company, New York, 1969.

[15] Laws, J. O,. and D. A. Parsons, The Relation of Raindrops Size to Intensity, Trans. Am. Geophysical Union, Vol. 24, 1943, pp. 452-460.

[16] Rensch, D, B., and R. K. Long, Comparative Studies of Extinction and Backscattering By Aerosols, Fog and Rain at $10.6 \mu$ and $0.63 \mu$, App. Opt., Vol. 9, No. 7, July 1970, pp. 1563-1573.

[17] L W Barclay, Terahertz Propagation, Lancaster University, Book: Propagation of Radiowaves, Published Dec 2002

[18] Mourad Chabane, A new quality of service FSO software, Proc. SPIE, Vol. 5465, 180, 2004.

[19] Bloom, S. Eric Korevaar, John Schuster, Heinz Willebrand, Understanding the Performance of free space optics [Invited], Journal of Optical Networking, 2003. 